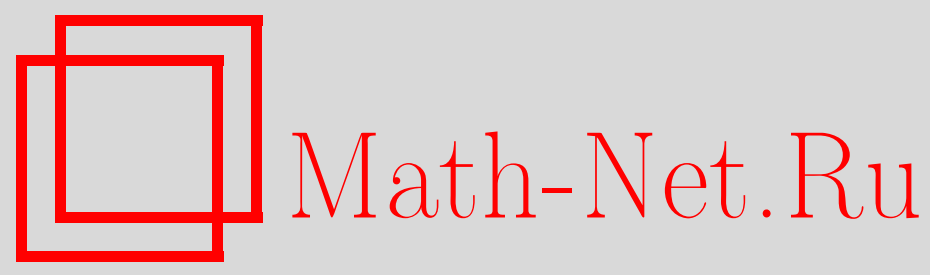

П. Л. Иванков, О линейной независимости некоторых чисел, Матем. заметки, 1997, том 62, выпуск 3, 383-390

DOI: https://doi.org/10.4213/mzm1620

Использование Общероссийского математического портала Math-Net.Ru подразумевает, что вы прочитали и согласны с пользовательским соглашением http://www. mathnet.ru/rus/agreement

Параметры загрузки:

IP : 54.198 .55 .26

26 апреля 2023 г., 10:18:21

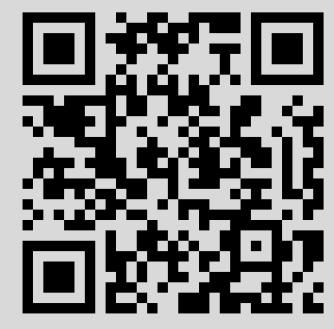




\title{
О ЛИНЕЙНОЙ НЕЗАВИСИМОСТИ НЕКОТОРЫХ ЧИСЕЛ
}

\section{П. Л. Иванков}

\begin{abstract}
В работе с помощью одного из вариантов метода Зигеля установлена линейная независимость значений гипергеометрических функций с иррациональньми параметрами и их производных в достаточно общей ситуации и получены соответствующие количественные результаты.

Библиограффия: 9 названий.
\end{abstract}

1. Две теоремы. Изучение арифметических свойств значений гипергеометрических функций обычно начинается с построения линейной приближающей формы, имеюшей при $z=0$ нуль достаточно высокого порядка. Метод построения такой формы основывается на применении принципа Дирихле или на использовании эффективной конструкции. В работе [1] предложен новьй подход к задаче построения приближающей формы, сочетающей в себе элементы обоих методов. В дальнейшем этот подход был использован в работе [2]. С помошью дополнительных соображений можно расширить область применимости указанного подхода.

Теорема 1. Пусть $a_{k}(x), b_{k}(x)$ - многочлень, все корни которых рациональны, а коэффициенты при стариих степенях равны $1 ; \operatorname{deg} a_{k}(x)=r_{k}, \operatorname{deg} b_{k}(x)=u_{k}$, $u_{k}-r_{k}=v_{k} \geqslant 0, k=1, \ldots, t ; d_{1}, \ldots, d_{t}$ - такие делители соответственно чисел $v_{1}, \ldots, v_{t}$, что $\lambda=v_{k} / d_{k}$ не зависит от $k$ (если $v_{k} \neq 0$, то можсно, например, положить $\left.d_{k}=v_{k}\right)$. Пусть далее, $b(x)=\left(x+\beta_{1}\right) \cdots\left(x+\beta_{u}\right)-$ многочлен скоэффициентами из некоторого мнимого квадратичного поля $\mathbb{I}, \beta_{1}, \ldots, \beta_{u}$ - алгебраические числа степеней соответственно $\varkappa_{1}, \ldots, \varkappa_{u}$,

$$
\tau=1-\frac{1}{u} \sum_{i=1}^{u} \frac{1}{\varkappa_{i}} .
$$

Предположим, что $r_{k}<m_{k}=u_{k}+d_{k} u, u$ рассмотрим при $k=1, \ldots, t$ функиии

$$
\psi_{k}(z)=\sum_{\nu=0}^{\infty} z^{d_{k} \nu} \omega_{k}^{d_{k} \nu} \prod_{x=1}^{\nu} \frac{a_{k}(x)}{b_{k}(x)} \prod_{x=1}^{d_{k} \nu} \frac{1}{b(x)},
$$

әде $\omega_{k}-$ числа из поля $\mathbb{I}$. Если функиии

$$
1, \psi_{k}^{\left(j_{k}\right)}(z), \quad k=1, \ldots, t, \quad j_{k}=0,1, \ldots, m_{k}-1,
$$

Работа выполнена при частичной поддержке Международного научного фонда, грант MHS000. 
линейно независимы над $\mathbb{C}(z)$, то для любого $\varepsilon>0$ и для любого нетривиального набора $h_{0}, h_{k j_{k}}-$ чельх чисел из поля $\mathbb{I}$ при $H=\max \left|h_{k j_{k}}\right|>H_{0}\left(\psi_{k}(z), \mathbb{I}(\varepsilon)\right)$ выполняется неравенство

$$
\left|h_{0}+\sum_{k=1}^{t} \sum_{j_{k}=0}^{m_{k}-1} h_{k j_{k}} \psi_{k}^{\left(j_{k}\right)}(1)\right|>H^{-\frac{m(\lambda+u)+u \tau}{\lambda+u-u \tau}-\varepsilon},
$$

əде $m=m_{1}+\cdots+m_{t}$.

Если в сформулированной теореме положить $a_{k}(x) \equiv 1, u_{1}=\cdots=u_{t}, d_{1}=\cdots=$ $d_{t}=1$, то получится первое утверждение теоремы из работы [1], а если $v_{1}=\cdots=v_{t}$, $d_{1}=\cdots=d_{t}=1$, то получится теорема 1 из [3].

ТЕОРема 2. Пусть выполнены все условия предыдущей теоремы,

$$
a_{k}(x) \equiv 1, \quad b_{k}(0)=0, \quad k=1, \ldots, t .
$$

Относительно функиий $\psi_{k}^{\left(j_{k}\right)}(z), k=1, \ldots, t, j_{k}=0,1, \ldots, m_{k}-1$, предположим, что они линейно независимы над $\mathbb{C}(z)$. Тогда для любого нетривиального набора $h_{k j_{k}}-$ чельх чисел из поля II при $H=\max \left|h_{k j_{k}}\right| \geqslant 3$ выполняется неравенство

$$
\left|\sum_{k=1}^{t} \sum_{j_{k}=0}^{m_{k}-1} h_{k j_{k}} \psi_{k}^{\left(j_{k}\right)}(1)\right|>H^{1-m-\frac{\gamma}{\sqrt{\ln \ln H}}},
$$

әде $\gamma>0$ - постоянная, зависящая от параметров функиий $\psi_{k}(z)$ и от поля $\mathbb{I}$ : $m=m_{1}+\cdots+m_{t}$.

Последняя теорема обобщает второе утверждение теоремы из [1]. Заметим еще, что для исследования арифметической природы значений функции (1) нельзя непосредственно применить метод Зигеля (см. [4, гл. 3]), так как эти функции не сводятся к $E$-функциям (см. по этому поводу работу работу [5]).

2. Доказательство теоремы 1. Через $\gamma_{1}, \gamma_{2}, \ldots$ будем обозначать положительные постоянные, зависящие от функций $\psi_{1}(z), \ldots, \psi_{t}(z)$ и от поля $\mathbb{I}$. При $0<\varepsilon<1$ положим $N_{\varepsilon}=[(1-\varepsilon) n / m], n-$ натуральньй параметр.

ЛЕмма 1. Для любого $\varepsilon, 0<\varepsilon<1$, при достаточно большом $n$ существует такой нетривиальный набор $y_{s}, s=0,1, \ldots, n$, иелых чисел из поля $\mathbb{I}$, по модулю не превосходящих $n^{\varepsilon n}$, что

$$
\begin{aligned}
& \sum_{s \equiv g_{k}\left(d_{k}\right)} \frac{y_{s}}{\omega_{k}^{s}}\left(\frac{n !}{s !}\right)^{\lambda} \prod_{N_{\varepsilon}+1 \leqslant d_{k} \leqslant N_{\varepsilon}+n-s} \frac{1}{b_{k}(x)} \\
& \times \prod_{d_{k} \leqslant d_{k}} \prod_{k \leqslant n+1-s} a_{k}(x) \frac{s(s-1) \cdots\left(s-\mu_{k}+1\right)}{\mu_{k} !}=0 \\
& g_{k}=0,1, \ldots, d_{k}-1, \quad \mu_{k}=0,1, \ldots, M_{k}, \quad M_{k}=\left[2 m_{k}+\frac{\left(N_{\varepsilon}-1\right) m_{k}}{d_{k}}-1\right] \\
& k=1, \ldots, t,
\end{aligned}
$$

где сумма распространена на все $s, 0 \leqslant s \leqslant n$, сравнимые $с g_{k}$ по модулю $d_{k}$, а произведения - на все целье неотрицательные значения $x$, удовлетворяющие указанным ограничениям. 
ДокАЗАТЕЛЬСтво леммы проводится стандартным способом. Рассматривая равенства (3) как систему линейных однородных уравнений относительно $y_{s}$, нетрудно установить, что общий наименьший знаменатель и абсолютная величина коэффищиентов этой системы растут при $n \rightarrow \infty$ медленнее любой положительной степени выражения $n^{n}$; число уравнений в системе не превосходит $\gamma_{1}+(1-\varepsilon) n$. Из этих фактов легко выводится существование чисел $y_{s}$ с требуемьпи свойствами (см., например, доказательство леммы 11 из [4, с. 109]).

Пусть

$$
\begin{gathered}
\psi_{k j_{k}}(z)=\sum_{\nu=0}^{\infty} z^{d_{k} \nu} \omega_{k}^{d_{k} \nu} \nu^{j_{k}-1} \prod_{x=1}^{\nu} \frac{a_{k}(x)}{b_{k}(x)} \prod_{x=1}^{d_{k} \nu} \frac{1}{b(x)}, \\
p_{s}=y_{s}\left(\frac{n !}{s !}\right)^{\lambda} \prod_{x=N_{\varepsilon}+1}^{N_{\varepsilon}+n-s} b(x), \quad s=0,1, \ldots, n, \\
P(z)=\sum_{s=0}^{n} p_{s} z^{s}, \quad P(z) \psi_{k j_{k}}(z)=\sum_{\nu=0}^{\infty} w_{k j_{k} \nu} z^{\nu}, \quad j_{k}=1, \ldots, m_{k}, \quad k=1, \ldots, t .
\end{gathered}
$$

ЛЕмма 2. Справедливь равенства $w_{k j_{k} \nu}=0, k=1, \ldots, t, j_{k}=1, \ldots, m_{k}$, $\nu=n+1, \ldots, n+N_{\varepsilon}$.

ДокАЗАТЕЛЬСтво. Имеем

$$
\begin{aligned}
w_{k j_{k} \nu}= & \sum_{s \equiv \nu\left(d_{k}\right)} p_{s} \omega_{k}^{\nu-s}\left(\frac{\nu-s}{d_{k}}\right)^{j_{k}-1} \prod_{x=1}^{(\nu-s) / d_{k}} \frac{a_{k}(x)}{b_{k}(x)} \prod_{x=1}^{\nu-s} \frac{1}{b(x)} \\
= & \omega_{k}^{\nu} \prod_{x=1}^{N_{\varepsilon}} \frac{1}{b(x)} \prod_{d_{k} \leqslant d_{k} x \leqslant N_{\varepsilon}} \frac{1}{b_{k}(x)} \sum_{s \equiv \nu\left(d_{k}\right)} \frac{y_{s}}{\omega_{k}^{s}}\left(\frac{n !}{s !}\right)^{\lambda} \\
& \times \prod_{k}(x) \\
& \times \prod_{d_{k} \leqslant d_{k} x \leqslant n+1-s} a_{k}\left(\frac{\nu-s}{d_{k}}-x_{1}\right) \prod_{x_{2}=1}^{X_{1}}\left(\frac{\nu-s}{x_{k}}\right)^{j_{k}-1}\left(\frac{\nu-s}{d_{k}}+x_{k}\right)^{\prod_{k} \leqslant n+N_{\varepsilon}-s} \prod_{x=\nu+1}^{n+N_{\varepsilon}} b(x-s),
\end{aligned}
$$

где $X_{1}=-\left[(n+1-\nu) / d_{k}\right]-1, X_{2}=\left[\left(n+N_{\varepsilon}-\nu\right) / d_{k}\right]$ (по поводу сумм и произведений см. замечание в формулировке леммы 1$)$.

Мы видим, что в каждое слагаемое входит многочлен от символа $s$ (образованный множителем $\left((\nu-s) / d_{k}\right)^{j_{k}-1}$ и произведениями по $x_{1}$ и $\left.x_{2}\right)$. Степень его не превосходит, очевидно, $M_{k}$, поэтому его можно представить в виде

$$
\sum_{\mu_{k}=0}^{M_{k}} \theta_{\mu_{k}} \frac{s(s-1) \cdots\left(s-\mu_{k}+1\right)}{\mu_{k} !}
$$


с некоторыми коэффициентами $\theta_{\mu_{k}}$. Выберем теперь число $g_{k}, 0 \leqslant g_{k} \leqslant d_{k}-1$, сравнимое с $\nu$ по модулю $d_{k}$, умножим равенство (3), отвечающее выбранному $g_{k}$ и числу $\mu_{k}$, на $\theta_{\mu_{k}}$ и сложим все получившиеся равенства (при выбранном $g_{k}$ и $\mu_{k}=0,1, \ldots, M_{k}$ ). Левая часть полученного таким способом равенства будет с точностью до вьнесенного за знак суммы множителя совпадать с правой частью равенства (4), а правая часть будет равна нулю. Из предположения о линейной независимости функций $1, \psi_{k j_{k}}(z)$ следует, что $\omega_{k} \neq 0, k=1, \ldots, t$. Поэтому сумма из правой части равенства (4) равна нулю, а тогда и лемма 2 доказана.

Обозначим

$$
\begin{aligned}
P_{k j_{k}}(z) & =\sum_{\nu=0}^{n} w_{k j_{k} \nu} z^{\nu}, \\
R_{k j_{k}}(z) & =P(z) \psi_{k j_{k}}(z)-P_{k j_{k}}(z),
\end{aligned} \quad k=1, \ldots, t, \quad j_{k}=1, \ldots, m_{k} .
$$

Таким образом, мы получаем совместные приближения для совокупности функций $\psi_{k j_{k}}(z)$. Пользуясь тем, что эти функции удовлетворяют системе линейных дифференциальных уравнений, построим целую совокупность таких совместных приближений с отличным от тождественного нуля определителем с помощью метода, предложенного в работе [6]. Затем, повторив соответствующие рассуждения указанной работы, перейдем от функциональных приближающих форм к числовым. Получающийся при этом результат сформулируем в виде леммы.

ЛЕмма 3. Для любого $\varepsilon>0$ при всех достаточно больиих $n$ существует набор чисел $q_{l k j_{k}}, q_{l}, l=0,1, \ldots, m, k=1, \ldots, t, j_{k}=1, \ldots, m_{k}$, из поля $\mathbb{I}$, обладающий следуюшими свойствами:

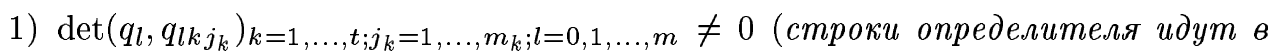
порядке возрастания $l$, а столбиы - в порядке возрастания $k$ и в порядке возрастания $j_{k}$ при равных $\left.k\right)$;

2) $\left|q_{l}\right| \leqslant n^{(\lambda+u+\varepsilon) n}$

3) $\left|q_{l} \psi_{k j_{k}}(1)-q_{l k j_{k}}\right| \leqslant n^{-((\lambda+u) / m-\varepsilon) n}, l=0,1, \ldots, m, k=1, \ldots, t, j_{k}=1, \ldots, m_{k}$;

4) числа $q_{l}$ являются иелыми в поле $\mathbb{I}$;

5) существует отличное от нуля челое число $G$ из поля $\mathbb{I}$ такое, что все числа $G q_{l k j_{k}}, l=0,1, \ldots, m, k=1, \ldots, t, j_{k}=1, \ldots, m_{k}$, являются иельми в поле $\mathbb{I}, u|G| \leqslant n^{(u \tau / m+\varepsilon) n}$.

С помощью леммы 3 можно стандартным способом (см., например, соответствующее место работы [6]) оценить снизу модуль произвольной линейной формы от чисел $\psi_{k j}(z)$, а отсюда уже нетрудно получить утверждение теоремы 1.

3. Доказательство теоремы 2. Нам потребуется ряд вспомогательных утверждений.

ЛЕмма 4. При любом достаточно большом натуральном $п$ существует такой нетривиальный набор иельх чисел из поля $\mathbb{I}$

$$
\begin{gathered}
y_{k g_{k} \mu_{k}}, \quad k=1, \ldots, t, \quad g_{k}=0,1, \ldots, d_{k}-1 \\
\mu_{k}=0,1, \ldots, M_{k}, \quad M_{k}=\left[\frac{m_{k} n}{d_{k}}-m_{k}-1\right]
\end{gathered}
$$


что при $0 \leqslant \nu \leqslant m n-n / \sqrt{\ln n}-1, g_{k} \equiv \nu\left(d_{k}\right), k=1, \ldots, t$, выполняется равенство

$$
\sum_{k=1}^{t} \omega_{k}^{\nu} \prod_{x=1}^{\left[\nu / d_{k}\right]} \frac{1}{b_{k}(x)} \sum_{\mu_{k}=0}^{M_{k}-g_{k}} y_{k g_{k} \mu_{k}} \frac{\nu(\nu-1) \cdots\left(\nu-\mu_{k}+1\right)}{\mu_{k} !} \prod_{x=1}^{g_{k}} b\left(\nu-g_{k}+x\right)=0
$$

$\Pi р и$ этом

$$
\left|y_{k g_{k} \mu_{k}}\right| \leqslant e^{\gamma_{2} n \sqrt{\ln n}}
$$

ДокАЗАТЕЛьство. Умножим каждое из равенств $(5)$ на $(\nu !)^{\lambda}$ и будем рассматривать эти равенства как систему линейных однородных уравнений относительно неизвестных $y_{k g_{k}} \mu_{k}$. Общий наименьший знаменатель коэффициентов такой системы и модули этих коэффициентов ограничены сверху величиной $e^{\gamma_{3} n}$ (при доказательстве используется рациональность корней многочленов $b_{k}(x)$; см., например, лемму 2 из [4, с. 186]). Количество уравнений в системе не превосходит, очевидно, $m n-n / \sqrt{\ln n}$, количество неизвестных не меньше $m n-\gamma_{4}$. Применяя [4, гл. 3 , лемма 13], легко завершаем доказательство леммы. Лемма 4 доказана.

Лемма 5. Для любого $k, 1 \leqslant k \leqslant t, u$ для любого $g_{k}, 0 \leqslant g_{k} \leqslant d_{k}-1$, существует $p_{k j_{k} s}$ - набор чисел из поля $\mathbb{I}\left(j_{k}=1, \ldots, m_{k}\right.$, а s пробегает все числа мнохсества $0,1, \ldots, n$, сравнимые $с g_{k}$ по модулю $d_{k}$ ) такой, что тохсдственно по $\nu$ выполняется равенство

$$
\begin{aligned}
& (n !)^{\lambda+u} \sum_{\mu_{k}=0}^{M_{k}-g_{k}} y_{k g_{k} \mu_{k}} \frac{\nu(\nu-1) \cdots\left(\nu-\mu_{k}+1\right)}{\mu_{k} !} \prod_{x=1}^{g_{k}} b\left(\nu-g_{k}+x\right) \\
& =\sum_{s \equiv g_{k}\left(d_{k}\right)} \sum_{j_{k}=1}^{m_{k}} p_{k j_{k} s}\left(\frac{\nu-s}{d_{k}}\right)^{j_{k}-1} \prod_{x=1}^{\left[s / d_{k}\right]} b_{k}\left(\frac{\nu-s}{d_{k}}+x\right) \prod_{x=1}^{s} b(\nu-s+x) .
\end{aligned}
$$

При этом при всех допустимых значениях индексов выполняется неравенство

$$
\left|p_{k j_{k} s}\right| \leqslant\left(\frac{n !}{s !}\right)^{\lambda+u} e^{\gamma_{5} n \sqrt{\ln n}}
$$

а общий наименьший знаменатель чисел $p_{k j_{k} s}$ ограничен сверху величиной $e^{\gamma_{6} n}$.

ДокАЗАТЕЛЬство. Зафиксируем $k$ и $g_{k}$, сократим обе части равенства (6) на общий множитель

$$
\prod_{x=1}^{g_{k}} b\left(\nu-g_{k}+x\right)
$$

и в получившееся равенство подставим столько различных натуральных значений $\nu$, сколько имеется чисел $p_{k j_{k} s}$. Получим систему линейных уравнений, которой должны удовлетворять числа $p_{k j_{k} s}$. Определитель этой системы отличен от нуля (он легко сводится к определителю Вандермонда). Отсюда следует, что числа, для которых равенство (6) вьполняется тождественно по $\nu$, существуют, определяются этим равенством 
однозначно и лежат в поле $\mathbb{I}$. Рассмотрим теперь совокупность многочленов от $z$ первой степени

$$
\begin{gathered}
1, \frac{z}{d_{k}}+\beta_{k 1}, \ldots, \frac{z}{d_{k}}+\beta_{k u_{k}}, \\
z+\beta_{1}, z+\beta_{1}-1, \ldots, z+\beta_{1}-d_{k}+1, \ldots, z+\beta_{u}, z+\beta_{u}-1, \ldots, z+\beta_{u}-d_{k}+1
\end{gathered}
$$

$\left(-\beta_{k 1}, \ldots,-\beta_{k u_{k}}-\right.$ корни многочлена $\left.b_{k}(x)\right)$. Занумеруем их в каком-нибудь порядке и обозначим через $\sigma_{1}(z), \ldots, \sigma_{m_{k}+1}(z)$, где $m_{k}=u_{k}+d_{k} u$. Определим, далее, многочлены

$$
\varkappa_{k j_{k}}(z)=\prod_{l=1}^{j_{k}-1} \sigma_{l}(z), \quad j_{k}=1, \ldots, m_{k}+1
$$

и запишем равенство, получающееся из (6) заменой $\left((\nu-s) / d_{k}\right)^{j_{k}-1}$ на $\varkappa_{k j_{k}}(\nu-s)$, $p_{k j_{k} s}$ на $p_{k j_{k} s}^{\prime}$ и сокрашением на указанный вьше обший множитель. Легко видеть, что правая часть полученного таким способом равенства есть разложение многочлена от $\nu$

$$
\varphi(\nu)=(n !)^{\lambda+u} \sum_{\mu_{k}=0}^{M_{k}-g_{k}} y_{k g_{k} \mu_{k}} \frac{\nu(\nu-1) \cdots\left(\nu-\mu_{k}+1\right)}{\mu_{k} !}
$$

в ряд Ньютона (см., например, 2.2 главы 2 из [7]). Поэтому числа $p_{k j_{k} s}^{\prime}$ определяются однозначно и могут быть записаны в виде контурных интегралов

$$
p_{k j_{k} s}^{\prime}=\frac{1}{2 \pi i} \oint_{\Gamma} \frac{\varphi(\zeta)}{\varkappa_{j_{k}+1}\left((\zeta-s) / d_{k}\right)} \frac{1}{\prod_{x=1}^{\left[s / d_{k}\right]} b_{k}\left((\zeta-s) / d_{k}+x\right)} \prod_{x=1}^{s} \frac{1}{b(\zeta-s+x)} d \zeta
$$

где контур интегрирования Г охватьвает все особые точки подынтегральной функции. Числа $p_{k j_{k} s}$ легко выражаются через числа $p_{k j_{k} s}^{\prime}$, а отсюда уже без труда можно вывести оставшиеся утверждения леммы; при этом для оценки общего наименьшего знаменателя чисел $p_{k j_{k} s}$ достаточно заметить, что интеграл из правой части равенства (7) равен (с точностью до знака) вычету подьштегральной функции относительно $\zeta=\infty$. Лемма 5 доказана.

Пусть

$$
P_{k j_{k}}(z)=\sum_{s=0}^{n} \omega_{k}^{s} p_{k j_{k} s} z^{s}
$$

где числа $p_{k j_{k} s}$ определены в предыдущей лемме.

ЛЕмма 6. Линейная форма

$$
R(z)=\sum_{k=1}^{t} \sum_{j_{k}=1}^{m_{k}} P_{k j_{k}}(z) \psi_{k j_{k}}(z)
$$

имеет при $z=0$ порядок нуля не меньше $m n-n / \sqrt{\ln n}$. 
ДокАЗАТЕЛЬСТво. Пусть

$$
R(z)=\sum_{\nu=0}^{\infty} w_{\nu} z^{\nu}
$$

Зафиксируем $\nu \geqslant 0$ и рассмотрим коэффициент $w_{\nu}$ при $z^{\nu}$. Имеем

$$
w_{\nu}=\sum_{k=1}^{t} \sum_{j_{k}=1}^{m_{k}} \sum_{s_{k} \equiv \nu\left(d_{k}\right)} \omega_{k}^{s_{k}} p_{k j_{k} s} \omega_{k}^{\nu-s_{k}}\left(\frac{\nu-s}{d_{k}}\right)^{j_{k}-1} \prod_{x=1}^{\left(\nu-s_{k}\right) / d_{k}} \frac{1}{b_{k}(x)} \prod_{x=1}^{\nu-s_{k}} \frac{1}{b(x)},
$$

где сумма по $s_{k}$ распространена на все значения $s_{k}$ из множества $0,1, \ldots, \min (n, \nu)$, сравнимые с $\nu$ по модулю $d_{k}$. Пусть $\nu \equiv g_{k}\left(d_{k}\right), 0 \leqslant g_{k} \leqslant d_{k}-1, k=1, \ldots, t$. Преобразуем выражение для $w_{\nu}$ следующим образом

$$
\begin{aligned}
w_{\nu}= & \prod_{x=1}^{\nu} \frac{1}{b(x)} \sum_{k=1}^{t} \omega_{k}^{\nu} \prod_{x=1}^{\left[\nu / d_{k}\right]} \frac{1}{b_{k}(x)} \sum_{j_{k}=1}^{m_{k}} \sum_{s_{k}=g_{k}\left(d_{k}\right)} p_{k j_{k} s_{k}}\left(\frac{\nu-s_{k}}{d_{k}}\right)^{j_{k}-1} \\
& \times \prod_{x=1}^{\left[s_{k} / d_{k}\right]} b_{k}\left(\frac{\nu-s_{k}}{d_{k}}+x\right) \prod_{x=1}^{s_{k}} b\left(\nu-s_{k}+x\right)
\end{aligned}
$$

где без ограничения обшности можно считать, что индекс суммирования $s_{k}$ пробегает все числа множества $0,1, \ldots, n$, сравнимые с $g_{k}$ по модулю $d_{k}$, так как в силу условия $b_{k}(0)=0$ все слагаемые, отвечаюшие значениям $s_{k}$ большим $\nu$, равны нулю. По лемме 5 отсюда получаем, что $w_{\nu}$ с точностью до отличного от нуля множителя равно выражению

$$
\sum_{k=1}^{t} \omega_{k}^{\nu} \prod_{x=1}^{\left[\nu / d_{k}\right]} \frac{1}{b_{k}(x)} \sum_{\mu_{k}=0}^{M_{k}-g_{k}} y_{k g_{k} \mu_{k}} \frac{\nu(\nu-1) \cdots\left(\nu-\mu_{k}+1\right)}{\mu_{k} !} \prod_{x=1}^{g_{k}} b\left(\nu-g_{k}+x\right) .
$$

Последнее выражение равно нулю по лемме 4. Лемма 6 доказана.

С помощью лемм 4-6 нетрудно доказать теорему 2 , повторив с очевидными изменениями соответствующую часть доказательства теоремы 1 из [5, с. 411].

4. Приложения. Чтобы построить примеры на применение доказанных теорем, заметим прежде всего, что функции (1) можно записать в виде

$$
\psi_{k}(z)=\sum_{\nu=0}^{\infty} z^{d_{k} \nu}\left(d_{k}^{u} \omega_{k}^{d_{k}}\right)^{\nu} \prod_{x=1}^{\nu} \frac{a_{k}(x)}{B_{k}(x)}
$$

где

$$
B_{k}(x)=b_{k}(x) \prod_{l=0}^{d_{k}-1}\left(x+\frac{\beta_{1}-l}{d_{k}}\right) \cdots\left(x+\frac{\beta_{u}-l}{d_{k}}\right) .
$$

Далее, легко видеть, что если $q$ - натуральное число, то из линейной независимости над $\mathbb{C}(z)$ функций

$$
1, \psi_{k}^{\left(j_{k}\right)}\left(z^{q}\right), \quad k=1, \ldots, t, \quad j_{k}=0,1, \ldots, m_{k}-1,
$$

следует линейная независимость над $\mathbb{C}(z)$ функций (2). Пусть $q=\lambda+u$, где $\lambda$ определено в формулировке теоремы 1 . Тогда пример линейно независимой совокупности функций вида (8) можно построить с помошью теоремы 8 из [8, с. 227] (см. также [9]). Отсюда, как было указано выше, вытекает линейная независимость совокупности функций (2), и к этой совокупности можно применить доказанные теоремы. 


\section{СПИСОК ЦИТИРОВАННОЙ ЛИТЕРАТУРЫ}

[1] Галочкин А.И. О некотором аналоге метода Зигеля // Вестн. МГУ. Сер. 1. Матем., мех. 1986. № 2. C. $30-34$.

[2] Иванков П. Л. Об оценках некоторых линейных форм // Изв. вузов. Матем. 1993. № 2. C. $38-45$.

[3] Иванков П. Л. О линейной независимости значений целых гипергеометрических функций с иррациональными параметрами // Сиб. матем. ж. 1993. Т. 34. № 5. С. 53-62.

[4] Шидловский А. Б. Трансцендентные числа. М.: Наука, 1986.

[5] Галочкин А. И. О критерии принадлежности гипергеометрических функций Зигеля классу $E$-функций // Матем. заметки. 1981. Т. 29. № 1. С. 3-14.

[6] Chudnovsky D. V., Chudnovsky G. V. Applications of Padé approximation to Diophantine inequalities in values of $G$-functions // Lecture Notes in Math. 1985. V. 1135. P. 9-51.

[7] Фельдман Н. И. Седьмая проблема Гильберта. М.: Изд-во МГУ, 1982.

[8] Салихов В. Х. Формальные решения линейных дифференциальных уравнений и их применение в теории трансцендентных чисел // Тр. ММО. 1988. Т. 51. С. 223-256.

[9] Казаков В. В. О линейной неприводимости дифференциальных уравнений для гипергеометрических функций // Всесоюзная шпола "Конструктивные методы и алгоритмы теории чисел". Тезисы докл. Минск: Изд-во АН БССР, 1989. С. 65.

Московский государственный технический университет

Поступило

им. Н.Э. Баумана

09.11 .95 